\title{
Dual-Modality Immuno-PET and Near-Infrared Fluorescence Imaging of Pancreatic Cancer Using an Anti-Prostate Stem Cell Antigen Cys-Diabody
}

\author{
Kirstin A. Zettlitz ${ }^{1-3}$, Wen-Ting K. Tsai ${ }^{1-3}$, Scott M. Knowles ${ }^{1-3}$, Naoko Kobayashi ${ }^{3,4}$, Timothy R. Donahue ${ }^{2,3,5}$, \\ Robert E. Reiter ${ }^{3,4}$, and Anna M. Wu ${ }^{1-3}$ \\ ${ }^{1}$ Crump Institute for Molecular Imaging, UCLA, Los Angeles, California; ${ }^{2}$ Department of Molecular and Medical Pharmacology, \\ UCLA, Los Angeles, California; ${ }^{3}$ David Geffen School of Medicine, UCLA, Los Angeles, California; ${ }^{4}$ Department of Urology, UCLA, \\ Los Angeles, California; and ${ }^{5}$ Division of General Surgery, Department of Surgery, UCLA, Los Angeles, California
}

Pancreatic cancer has a high mortality rate due to late diagnosis and the tendency to invade surrounding tissues and metastasize at an early stage. A molecular imaging agent that enables both presurgery antigen-specific PET (immuno-PET) and intraoperative near-infrared fluorescence (NIRF) guidance might benefit diagnosis of pancreatic cancer, staging, and surgical resection, which remains the only curative treatment. Methods: We developed a dual-labeled probe based on A2 cys-diabody (A2cDb) targeting the cell-surface prostate stem cell antigen (PSCA), which is expressed in most pancreatic cancers. Maleimide-IRDye $800 \mathrm{CW}$ was site-specifically conjugated to the C-terminal cys-tag (A2cDb-800) without impairing integrity or affinity (half-maximal binding, $4.3 \mathrm{nM}$ ). Direct radioiodination with ${ }^{124}$ I ( $\left.{ }^{124} \mathrm{I}-\mathrm{A} 2 \mathrm{cDb}-800\right)$ yielded a specific activity of $159 \pm$ $48 \mathrm{MBq} / \mathrm{mg}$ with a radiochemical purity exceeding $99 \%$ and $65 \% \pm$ $4.5 \%$ immunoreactivity $(n=3)$. In vivo specificity for PSCA-expressing tumor cells and biodistribution of the dual-modality tracer were evaluated in a prostate cancer xenograft model and compared with single-labeled ${ }^{124} \mathrm{I}-\mathrm{A} 2 \mathrm{cDb}$. Patient-derived pancreatic ductal adenocarcinoma xenografts (PDX-PDACs) were grown subcutaneously in NSG mice and screened for PSCA expression by immuno-PET. Small-animal PET/CT scans of PDX-PDAC-bearing mice were obtained using the dual-modality ${ }^{124} \mathrm{I}-\mathrm{A} 2 \mathrm{cDb}-800$ followed by postmortem NIRF imaging with the skin removed. Tumors and organs were analyzed ex vivo to compare the relative fluorescent signals without obstruction by other organs. Results: Specific uptake in PSCA-positive tumors and low nonspecific background activity resulted in high-contrast immuno-PET images. Concurrent with the PET studies, fluorescent signal was observed in the PSCA-positive tumors of mice injected with the dual-tracer ${ }^{124} \mathrm{I}-\mathrm{A} 2 \mathrm{CDb}-800$, with low background uptake or autofluorescence in the surrounding tissue. Ex vivo biodistribution confirmed comparable tumor uptake of both ${ }^{124}$ I-A2cDb-800 and ${ }^{124}$ I-A2cDb. Conclusion: Dual-modality imaging using the anti-PSCA cys-diabody resulted in high-contrast immuno-PET/NIRF images of PDX-PDACs, suggesting that this imaging agent might offer both noninvasive whole-body imaging to localize PSCA-positive pancreatic cancer and fluorescence imageguided identification of tumor margins during surgery.

Received Dec. 21, 2017; revision accepted Mar. 12, 2018.

For correspondence or reprints contact: Kirstin A. Zettlitz, Crump Institute for Molecular Imaging, CNSI 4350B, 570 Westwood Plaza, Los Angeles, CA 90095.

E-mail: kzettlitz@mednet.ucla.edu

Guest Editor: David Mankoff, University of Pennsylvania

Published online Mar. 30, 2018.

COPYRIGHT @ 2018 by the Society of Nuclear Medicine and Molecular Imaging.
Key Words: immuno-PET; fluorescence-guided surgery; cys-diabody; prostate stem cell antigen (PSCA); pancreatic cancer

J Nucl Med 2018; 59:1398-1405

DOI: $10.2967 /$ jnumed.117.207332

$\mathbf{P}$ ancreatic ductal adenocarcinoma, the most common form of pancreatic cancer ( $85 \%$ of cases), is usually diagnosed at an advanced, incurable stage and is resistant to therapy, making it the most lethal common cancer (1). A combination of medical imaging techniques, such as contrast-enhanced CT and endoscopic ultrasound, is currently used to confirm diagnosis and categorize the resectability of pancreatic cancer (2). After staging, only $15 \%-$ $20 \%$ of patients are candidates for surgical resection, which remains the only potentially curative treatment. The presence of undetected metastatic disease, and the high rate of positive lymph nodes and incomplete resection at the time of surgery, lead to the low 5-y survival $(10 \%-15 \%)$ of patients undergoing surgery with curative intent (2). Several studies support a role for PET $\left({ }^{18} \mathrm{~F}\right.$ FDG PET/CT), mostly in the initial management of known or suspected malignancy, and have shown ${ }^{18} \mathrm{~F}-\mathrm{FDG}$ PET/CT to be advantageous for the detection of distant metastases (3). However, differentiation between pancreatic carcinoma and mass-forming pancreatitis remains a diagnostic limitation of metabolic imaging because of ${ }^{18} \mathrm{~F}$-FDG accumulation in inflammatory processes (4).

The poor prognosis of pancreatic cancer, together with the lack of imaging methods adequate for early detection, explains the great need to develop new markers and imaging modalities. Improved tumor-specific imaging for more accurate staging (tumor size, local advancement into blood or lymphatic vessels and neighboring organs, spread to lymph nodes and metastasis) might help with the critically important assessment of resectability and avoid major surgery for patients unlikely to benefit. Real-time intraoperative imaging might help the surgeon see malignant cells that need to be removed (5) in order to ensure complete resection and lower local recurrence (occurs in 50\%-80\% of patients) $(6,7)$.

Prostate stem cell antigen (PSCA) is a 123-amino-acid glycosylphosphatidylinositol-anchored cell-surface glycoprotein expressed mostly in the prostate and with limited normal-tissue expression (8). PSCA is upregulated in virtually all prostate cancers $(83 \%-100 \%)$, and higher PSCA levels have been correlated with poor prognosis 
and metastatic disease (9-11). PSCA is also overexpressed in bladder and pancreatic carcinoma. Two studies reported PSCA overexpression in most primary pancreatic ductal adenocarcinoma samples; using immunohistochemistry, between 60\% (36/60) and 93\% (13/14) of primary tumor samples stained positively for PSCA and showed strong PSCA expression in the neoplastic epithelium and absence of PSCA in adjacent nonneoplastic pancreas (epithelium and stroma) $(12,13)$. Another study showed PSCA messenger RNA to be elevated in human pancreatic cancer tissues compared with chronic pancreatitis $(79 \%, 19 / 24)$ or normal pancreas $(88 \%$, 21/24) (14). The cell-surface expression and the ability to distinguish pancreatic cancer from chronic pancreatitis make PSCA a promising diagnostic and therapeutic target for antibody-based applications $(14,15)$.

Antibody-based dual-modality imaging enables both presurgery antigen-specific PET (immuno-PET) and intraoperative near-infrared fluorescence (NIRF) for image-guided surgery.

Immuno-PET uses the high specificity of antibodies in combination with the sensitivity of PET for noninvasive imaging of cellsurface biomarkers (16-18). Engineered antibody fragments, such as the diabody (single-chain variable fragment dimer, $50 \mathrm{kDa}$ ), have pharmacokinetics optimized for imaging, with fast renal clearance for high target-to-background ratios at early time points after administration. Removal of the Fc region abolishes effector function and neonatal Fc receptor recycling, and antibody fragments can be further modified to include sites for specific conjugation (19). Renal clearance is especially advantageous for imaging pancreatic cancer and for detecting liver metastasis. Although the short plasma halflife of diabodies would enable the application of shorter-lived PET tracers, using ${ }^{124}$ I (half-life, $4.2 \mathrm{~d}$ ) has several advantages. Direct iodination to surface-exposed tyrosine residues is effective and convenient, and several studies have shown minimal impact on plasma clearance of the radiolabeled antibody (20-22). On internalization and degradation of the antibody, ${ }^{124}$ I-iodide or ${ }^{124}$ I-iodotyrosine can diffuse from the cell, resulting in greatly reduced tissue background activity. PET resolution is intrinsically limited by the positron range (3 $\mathrm{mm}$ for $\left.{ }^{124} \mathrm{I}\right)$ and the resolution of the scanner (1.5-1.8 $\mathrm{mm}$ for small-animal PET) but provides whole-body evaluation with organlevel biodistribution. Another disadvantage is the time constraint due to the radionuclide half-life.

NIRF dyes, on the other hand, have no physical half-life, and the high spatial and temporal resolution of NIRF imaging is particularly useful for visualizing superficial tissue layers with cell-level resolution. Near-infrared dyes ( $\sim 700$ - to $1,000-\mathrm{nm}$ emission) show good tissue penetration (ranging from millimeters to $\sim 1.0 \mathrm{~cm}$ ), and most tissue exhibits minimal autofluorescence in the near-infrared spectrum, leading to high signal-to-background contrast (23). The availability of specialized intraoperative imaging systems has led to an increased interest in real-time fluorescence imaging guidance to benefit surgical resection and identification of tumor margins $(5,24)$. The NIRF dye IRDye800CW has clinical relevance because it is available with a variety of functionalities for antibody labeling and has been used in several clinical studies (25-27).

We developed a dual-labeled probe based on the humanized, affinity-matured anti-PSCA A2 cys-diabody (A2cDb) by sitespecific conjugation of IRDye800CW and random ${ }^{124}$ I-labeling ( $\left.{ }^{124} \mathrm{I}-\mathrm{A} 2 \mathrm{cDb}-800\right)$. The dual-modality imaging agent was evaluated by sequential immuno-PET and NIRF imaging of mice bearing prostate cancer xenografts with low and high PSCA expression and mice bearing patient-derived pancreatic ductal adenocarcinoma xenografts (PDX-PDACs).

\section{MATERIALS AND METHODS}

The protocols for animal studies were approved by the UCLA Animal Research Committee.

Sample collection from human subjects was approved by the UCLA Institutional Review Board, and written informed consent was given by all participating human subjects.

\section{Anti-PSCA Cys-Diabody 2B3 A2 (A2cDb)}

$\mathrm{A} 2 \mathrm{cDb}$ was generated by adding a C-terminal his-cys-tag $\left(-\mathrm{H}_{6}-\mathrm{GGC}\right)$ to the humanized and affinity-matured anti-PSCA 2B3 A2 diabody (28). Production and purification of $\mathrm{A} 2 \mathrm{cDb}$ were previously described (29).

\section{Cell Lines and Xenografts}

Athymic nude (JAX002019) and NSG (JAX005557) male mice (6-8 wk) were purchased from The Jackson Laboratory. CWR22Rv1 cells expressing low levels of endogenous PSCA were obtained from ATCC and cultured in RPMI 1640 supplemented with 10\% fetal bovine serum. The generation of cell line 22Rv1-PSCA by transduction using a PSCA-expressing lentivirus was previously described (15). Tumors were implanted by injecting $1 \times 10^{6}$ cells in $1: 1$ phosphatebuffered saline:Matrigel (BD Biosciences) subcutaneously in the shoulder region of male nude mice. Patient-derived xenografts were established in NSG mice from pancreatic ductal adenocarcinoma samples collected from patients undergoing pancreaticoduodenectomy (Donahue Lab, UCLA).

\section{Immunohistochemistry}

Formalin-fixed tumor tissues were paraffin-embedded and cut to a 4- to 5- $\mu \mathrm{m}$ thickness by the Translational Pathology Core Laboratory at UCLA. The slides were deparaffinized and rehydrated, followed by heat-induced epitope retrieval (sodium citrate buffer, pH 6.0, 30 min in a vegetable steamer) and incubation with $3 \%$ hydrogen peroxide at room temperature for $15 \mathrm{~min}$. After washing and blocking, the slides were incubated with the parental mouse anti-PSCA 1G8 IgG1 (1/100) overnight at $4^{\circ}$ C. A M.O.M. immunodetection kit (BMK-2202; Vector Laboratories) was used for detection.

\section{Site-Specific Conjugation of IRDye800CW Maleimide}

$\mathrm{A} 2 \mathrm{cDb}(30 \mu \mathrm{M}$ in $50 \mu \mathrm{L}$ of phosphate-buffered saline) was reduced by incubation with a 10-fold molar excess of tris(2-carboxyethyl)phosphine (Pierce) for $1 \mathrm{~h}$ at room temperature. A 5-fold molar excess of IRDye $800 \mathrm{CW}$ maleimide (LI-COR) was added and incubated overnight at $4^{\circ} \mathrm{C}$. Excess dye was removed using Micro Bio-Spin sizeexclusion columns (Bio-Rad). Size-exclusion chromatography was performed using a Superdex 75 HR 10/30 column (GE Healthcare) on an ÄKTApurifier (GE Healthcare) with phosphate-buffered saline as the running buffer $(0.5 \mathrm{~mL} / \mathrm{min})$. Absorbance at $280 \mathrm{~nm}$ for protein detection and at $700 \mathrm{~nm}$ for NIRF dye detection was recorded. Purified conjugates were analyzed by sodium dodecyl sulfate polyacrylamide gel electrophoresis and scanned on an Odyssey CLx (LI-COR).

\section{Flow Cytometry and Cell-Binding Affinity}

Cells (22Rv1-PSCA and 22Rv1, $1 \times 10^{5}$ ) were incubated with serial dilutions of A2cDb-IRDye800CW for $4 \mathrm{~h}$ at $4^{\circ} \mathrm{C}$, washed (phosphatebuffered saline, $1 \%$ fetal bovine serum), and analyzed using a FACScan system (BD Biosciences). Data were evaluated with FlowJo (version 9.3.2; BD Biosciences) and fitted with Prism (version 7.0b; GraphPad Software) to a single-site saturation binding model. The apparent affinity was calculated from 3 independent binding curves.

\section{Radioiodination}

${ }^{124}$ I-labeling of antibody fragments was done by direct iodination using Pierce precoated iodination tubes (Thermo Scientific) according to the manufacturer's instructions. One hundred micrograms of protein were incubated with $15 \mathrm{MBq}$ of $\mathrm{Na}^{124} \mathrm{I}$ (3D Imaging LLC) in $0.1 \mathrm{M}$ 


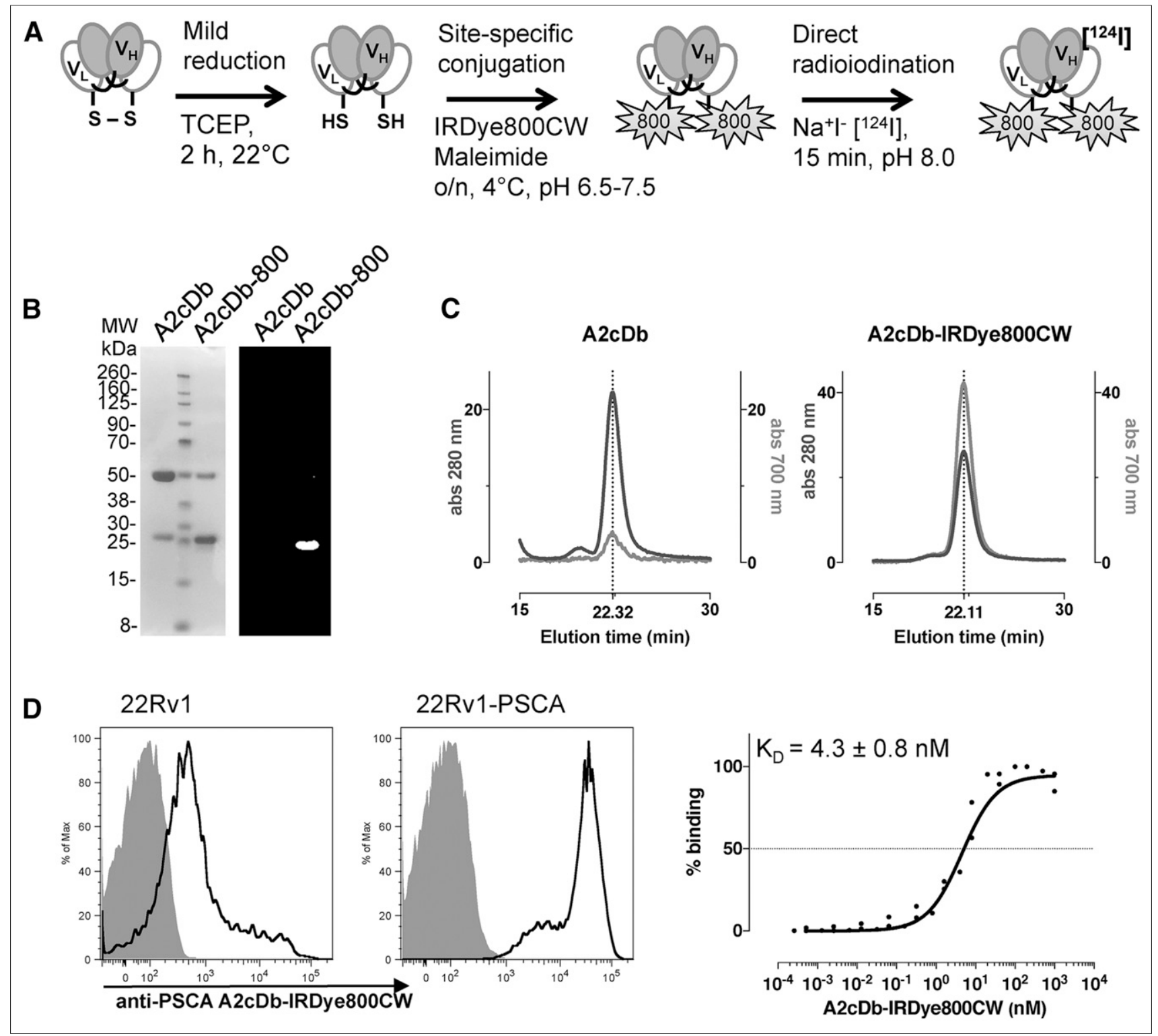

FIGURE 1. Biochemical characterization of A2cDb-IRDye800CW. (A) Schematic of dual-modality labeling. (B) Sodium dodecyl sulfate polyacrylamide gel electrophoresis analysis of unconjugated $\mathrm{A} 2 \mathrm{cDb}$ and site-specifically conjugated $\mathrm{A} 2 \mathrm{cDb}$-IRDye800 under nonreducing conditions: Coomassie-stained (left) and NIRF (right) images. (C) Size-exclusion chromatography showing elution profiles for protein (280 nm) and NIRF dye $(700 \mathrm{~nm})$. (D) Flow cytometry analysis of A2cDb-IRDye800 binding to cells expressing PSCA at low (22Rv1) and high (22Rv1-PSCA) levels (left), and titration of A2cDb-IRDye800 to calculate half-maximal binding $\left(\mathrm{K}_{\mathrm{D}}\right)$ using single-site specific binding model $(n=3$, GraphPad) (right). MW $=$ molecular weight; TCEP $=$ tris(2-carboxyethyl)phosphine; $\mathrm{V}_{\mathrm{H}}=$ heavy-chain variable domain; $\mathrm{V}_{\mathrm{L}}=$ light-chain variable domain.

tris, $\mathrm{pH}$ 8.0. Micro Bio-Spin size-exclusion columns were used for removal of free radiolabel and buffer exchange. Labeling efficiency and radiochemical purity were determined using instant thin-layer

TABLE 1

Radioiodination of $\mathrm{A} 2 \mathrm{cDb}$ and A2cDb-IRDye800CW $(n=3)$

\begin{tabular}{lcc}
\hline \multicolumn{1}{c}{ Parameter } & $124 \mathrm{I}-\mathrm{A} 2 \mathrm{cDb}$ & ${ }^{124} \mathrm{I}-\mathrm{A} 2 \mathrm{cDb}-800$ \\
\hline Labeling efficiency (\%) & $93.1 \pm 5.2$ & $91.8 \pm 6.8$ \\
\hline Specific activity $(\mathrm{MBq} / \mathrm{mg})$ & $155.4 \pm 33.3$ & $159.1 \pm 48.1$ \\
Radiochemical purity (\%) & $98.3 \pm 2.1$ & $99.5 \pm 0.3$ \\
Immunoreactivity (\%) & $75.6 \pm 3.4$ & $65.0 \pm 4.5$ \\
\hline
\end{tabular}

chromatography strips for monoclonal antibody preparation (Biodex Medical Systems) and saline as solvent. Immunoreactive fractions were determined by incubation of radiolabeled antibodies $(0.5 \mathrm{ng})$ with an excess of antigen-expressing cells (22Rv1-PSCA) or control cells (22Rv1). The cells were washed, and radioactivity associated with them or in the supernatant was measured by $\gamma$-counting (Wizard 3" 1480 automatic $\gamma$-counter; Perkin-Elmer).

\section{Immuno-PET/CT Imaging}

Thyroid and stomach uptake of radioiodine was blocked with Lugol iodine and potassium perchlorate, respectively, as previously described (30). Approximately $15 \mu \mathrm{g}$ (2.96 MBq) of ${ }^{124} \mathrm{I}-\mathrm{A} 2 \mathrm{cDb}$ or ${ }^{124} \mathrm{I}-\mathrm{A} 2 \mathrm{cDb}-$ 800 were injected via the tail vein into tumor-bearing mice. Twenty-four hours after injection, the mice were anesthetized using $1.5 \%$ isoflurane, and static 10-min small-animal PET acquisitions (Inveon; Siemens) 


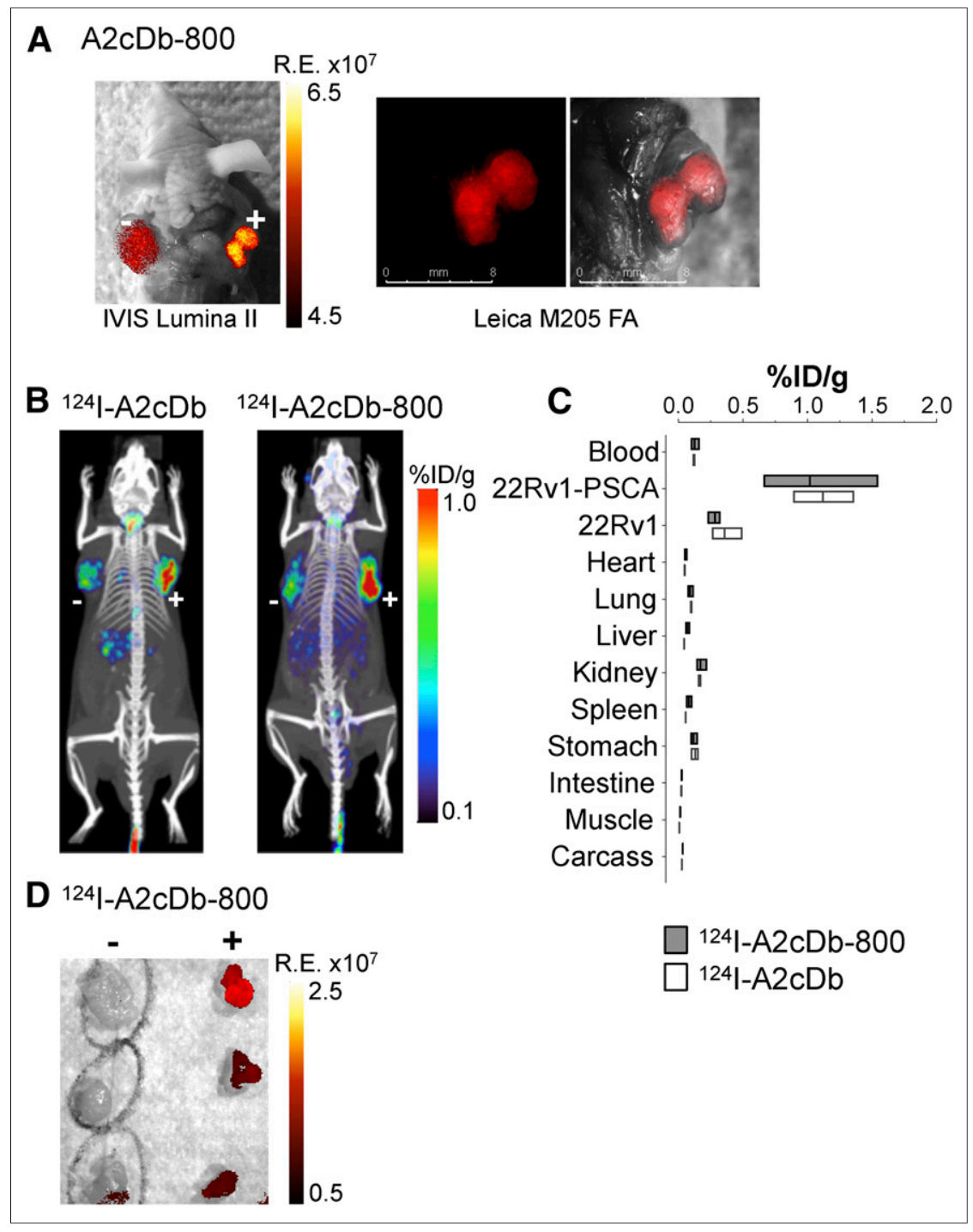

FIGURE 2. Dual-modality immuno-PET/NIRF imaging of PSCA-expressing prostate cancer xenografts. (A) A2cDb-IRDye800CW NIRF imaging of in situ tumors expressing PSCA at low (22Rv1, left shoulder) and high (22Rv1-PSCA, right shoulder) levels (postmortem imaging $2 \mathrm{~h}$ after injection, with skin removed) using IVIS Lumina II (left) and Leica M205 FA dissecting microscope (right). (B) Immuno-PET (24 h after injection) comparing ${ }^{124} \mathrm{I}-\mathrm{A} 2 \mathrm{cDb}$ and dual-modality ${ }^{124} \mathrm{I}-\mathrm{A} 2 \mathrm{cDb}-$ 800 in nude mice bearing 22Rv1 subcutaneous tumors expressing PSCA at low (left shoulder) and high (right shoulder) levels. Representative scans (of $n=3$ ) are depicted as whole-body maximum-intensity-projection small-animal PET/CT overlays. (C) Ex vivo biodistribution $24 \mathrm{~h}$ after injection $(n=3)$. (D) Postmortem optical imaging of mice that received dual-modality tracer ( $\left.{ }^{124} \mathrm{I}-\mathrm{A} 2 \mathrm{cDb}-800\right)$. Tumors were resected (22Rv1, left; 22Rv1-PSCA, right), and NIRF imaging was performed ex vivo. R.E. = radiant efficiency.

were followed by small-animal CT scans (CrumpCAT; UCLA in-house small-animal CT scanner). For ${ }^{124} \mathrm{I}-\mathrm{A} 11 \mathrm{Mb}$ immuno-PET, $35 \mu \mathrm{g}(2.96$ $\mathrm{MBq})$ were injected and the mice were scanned $48 \mathrm{~h}$ afterward $(30,31)$

The PET images were processed using non-attenuation-corrected filtered backprojection or maximum a posteriori reconstruction. PET/CT overlays were displayed as maximum-intensity projections of the whole body or 3-mm sections (coronal and transverse). Image analysis was performed using AMIDE (32).

\section{NIRF Imaging}

Postmortem optical imaging of intact mice with the skin removed, and ex vivo optical imaging of dissected tumors and organs, were done using an IVIS Lumina II system (PerkinElmer) with a 745-nm excitation wavelength, indocyanine green emission filter, and 1- to 10-s exposure time. Living Image software (PerkinElmer) was used to analyze and display fluorescence and visible-light overlays.

\section{Biodistribution}

Blood and tissues were collected, weighed, and $\gamma$-counted. The percentage injected dose per gram of tissue $(\% \mathrm{ID} / \mathrm{g})$ was calculated on the basis of standards containing $1 \%$ of the injected dose.

\section{Data Analysis}

Data are reported as mean \pm SD unless indicated otherwise. Ex vivo biodistribution values are shown as floating bar (minimum to maximum) graphs. For statistical analysis, paired $t$ tests were performed using Prism.

\section{RESULTS}

\section{Dual-Modality Anti-PSCA \\ Cys-Diabody}

The anti-PSCA cys-diabody $\mathrm{A} 2 \mathrm{cDb}$ was site-specifically conjugated with IRDye800CW-maleimide after reduction of the C-terminal cys-tag (A2cDb-800, Fig. 1A), resulting in a dye-to-protein ratio of $1.37 \pm 0.15(n=3)$. Sodium dodecyl sulfate polyacrylamide gel electrophoresis analysis and fluorescence imaging of the gel confirmed successful conjugation, with the fluorophore bound to the monomeric protein (migrating at $25 \mathrm{kDa}$, Fig. 1B). Size-exclusion chromatography showed similar elution profiles for the unconjugated $\mathrm{A} 2 \mathrm{cDb}$ and $\mathrm{A} 2 \mathrm{cDb}-\mathrm{IRD} y e 800 \mathrm{CW}$, verifying that the dimeric conformation of the cys-diabody was not impaired by breaking the interchain disulfide bond and NIRF dye conjugation (Fig. 1C). The elution profile for the NIRF dye $(700 \mathrm{~nm})$ was concurrent with the profile for protein $(280$ $\mathrm{nm}$ ), further corroborating successful conjugation. Specific binding of $\mathrm{A} 2 \mathrm{cDb}-800$ to antigen-expressing cells was demonstrated by flow cytometric analysis using a prostate cancer cell line expressing minimal levels of endogenous PSCA (22Rv1) and the same cell line transduced to express high levels of PSCA (22Rv1-PSCA, $2.2 \times 10^{6}$ antigens per cell) (31). Saturation binding of A2cDb-800 to 22Rv1-PSCA cells confirmed that the dye-conjugated protein retained low nanomolar affinity (half-maximal binding, $4.3 \pm 0.8 \mathrm{nM}, n=3$ ) (Fig. 1D).

$\mathrm{A} 2 \mathrm{cDb}$ and $\mathrm{A} 2 \mathrm{cDb}-800$ were radiolabeled by direct iodination, resulting in ${ }^{124} \mathrm{I}-\mathrm{A} 2 \mathrm{cDb}$ and the dual-modality tracer ${ }^{124} \mathrm{I}-\mathrm{A} 2 \mathrm{cDb}-$ 800 (Fig. 1A). Radioiodination was conducted with comparable labeling efficiencies, resulting in similar specific activities and immunoreactive fractions (Table 1). 
In Vivo Dual-Modality Imaging of PSCA-Expressing Prostate Cancer Xenografts

Specific antigen targeting in vivo was first shown for the NIRF dye-conjugated A2cDb-800. 22Rv1 cells with low and high PSCA expression were implanted into the opposing shoulders of male nude mice. Fluorescence imaging as early as $2 \mathrm{~h}$ after injection using the IVIS Lumina II system showed antigen-specific uptake in the 22Rv1-PSCA tumor (Fig. 2A). The fluorescent signal clearly distinguished the PSCA-expressing tumor from surrounding tissue and was visibly higher than in the $22 \mathrm{Rv} 1$ tumor. The same mouse was assessed in a surgical setting using a Leica M205 FA dissecting microscope (Fig. 2A). The resolution was high enough at the tissue level to enable identification of positive tumor margins during resection.

Whole-body distribution was analyzed by immuno-PET imaging $24 \mathrm{~h}$ after injection of the single-labeled ${ }^{124} \mathrm{I}-\mathrm{A} 2 \mathrm{cDb}$ or the dual-labeled ${ }^{124} \mathrm{I}-\mathrm{A} 2 \mathrm{cDb}-800$. Both tracers showed similar PSCAspecific tumor uptake and low activity in background tissues (Fig. 2B). 22Rv1 and 22Rv1-PSCA tumors resected from mice injected with the dual-modality probe ${ }^{124} \mathrm{I}-\mathrm{A} 2 \mathrm{cDb}-800$ were analyzed ex vivo to compare the relative fluorescent signals (Fig. 2D). Consistent with the immuno-PET results, high-contrast fluorescence was observed in the PSCA-expressing tumors (Fig. 2D). Ex vivo biodistribution confirmed that tumors with high PSCA expression (22Rv1-PSCA) were the tissue with the highest uptake $\left(1.1 \pm 0.2\right.$ and $1.0 \pm 0.5 \% \mathrm{ID} / \mathrm{g}$ for ${ }^{124} \mathrm{I}-\mathrm{A} 2 \mathrm{cDb}$ and ${ }^{124} \mathrm{I}-\mathrm{A} 2 \mathrm{cDb}-$ 800 , respectively) and very low remaining blood activity $(0.1$ $\% \mathrm{ID} / \mathrm{g}$ for both), resulting in similar positive tumor-to-blood ratios of 9.3 and 8.5, respectively (Fig. 2C; Supplemental Table 1; supplemental materials are available at http://jnm. snmjournals.org). Importantly, no significant difference in biodistribution or clearance was detected between single- and dualmodality $\mathrm{A} 2 \mathrm{cDb}$.

\section{I-Immuno-PET Imaging of PDX-PDACs}

PDX-PDACs implanted subcutaneously into the shoulder or flank of NSG mice were initially imaged using ${ }^{124}$ I-labeled antiPSCA minibody (A11 Mb, Fig. 3A). The higher molecular weight and longer plasma half-life of the minibody $(80 \mathrm{kDa})$ results in higher tumor uptake, ensuring visualization of xenografts expressing low levels of PSCA. Immuno-PET scans were acquired $48 \mathrm{~h}$ after injection, and all tested PDX-PDACs showed specific tracer uptake, although at different levels (Fig. 3A). Minimal background activity was observed in the stomach, probably because of incomplete blockage of the sodium-iodide symporter in the gastric mucosa. PSCA expression was confirmed by flow cytometry and immunohistochemistry with the parental anti-PSCA antibody 1G8 (Fig. 3C).

Even though the smaller antibody fragment cys-diabody $(\mathrm{A} 2 \mathrm{cDb})$ might reach lower tumor uptake values, its shorter half-life results in high tumor-to-background ratios at earlier time points, and the renal clearance of this antibody fragment would be beneficial for imaging the pancreas and liver. Two additional PDX-PDACs (XRW6 and XRW26) were imaged $24 \mathrm{~h}$ after injection of ${ }^{124} \mathrm{I}-\mathrm{A} 2 \mathrm{cDb}$, and high-contrast images were obtained (Fig. 3B). XRW26 xenografts took more than 4 mo to reach tumor sizes of 70-300 mg, and calcification was visible within the xenografts on CT scans $(n=4)$. Calcification has been reported for a variety of pancreatic neoplasms (33). XRW26 showed heterogeneous tracer uptake, with parts of the tumor reaching more than $5 \% \mathrm{ID} / \mathrm{g}$ and areas with calcification

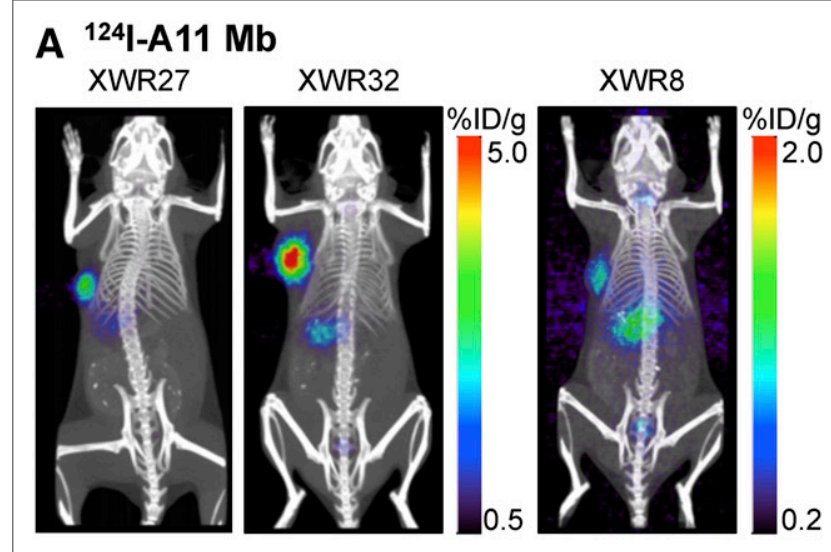

\section{B ${ }^{124} \mid-A 2 c D b$ XWR6}
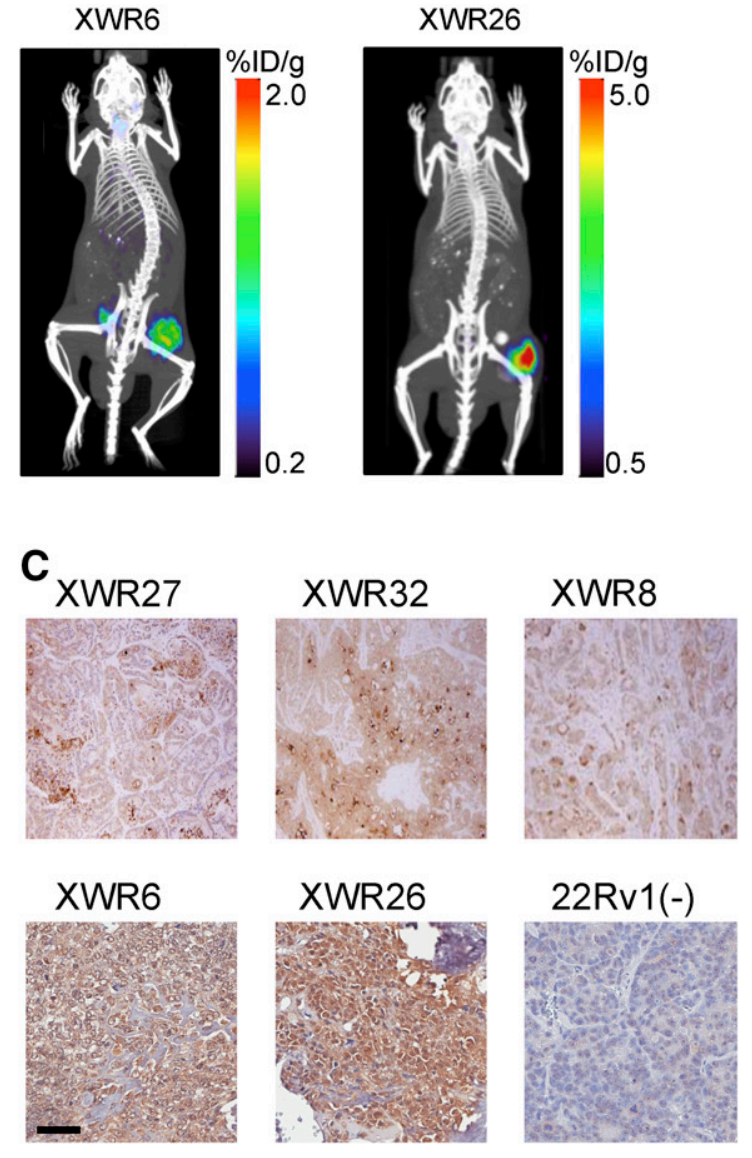

FIGURE 3. ${ }^{124}$-immuno-PET of PDX-PDACs. (A) ${ }^{124}$-A11 minibody (A11 Mb, $80 \mathrm{kDa}$ ) immuno-PET (35 $\mu \mathrm{g} / 2.96 \mathrm{MBq}$; $48 \mathrm{~h}$ after injection) of subcutaneous PDX-PDAC (XWR, left shoulder). (B) ${ }^{124} \mathrm{l}-\mathrm{A} 2 \mathrm{cDb}$ immuno-PET (15 $\mu \mathrm{g} / 2.96 \mathrm{MBq} ; 24 \mathrm{~h}$ after injection) of subcutaneous PDX-PDAC (XWR, right flank). (C) Immunohistochemical staining for PSCA in PDX-PDAC XWR using parental anti-PSCA antibody $1 \mathrm{G} 8(\times 40)$.

showing no uptake. Uptake in XWR6 tumors, at $0.9 \pm 0.2 \%$ $\mathrm{ID} / \mathrm{g}$, was lower but more uniform and provided excellent contrast (tumor-to-blood ratio, $10 \pm 1.8$; tumor-to-muscle ratio, $155 \pm 21[n=7]$; Supplemental Table 2). XWR6 was chosen for the subsequent evaluation of the dual-modality imaging approach. 


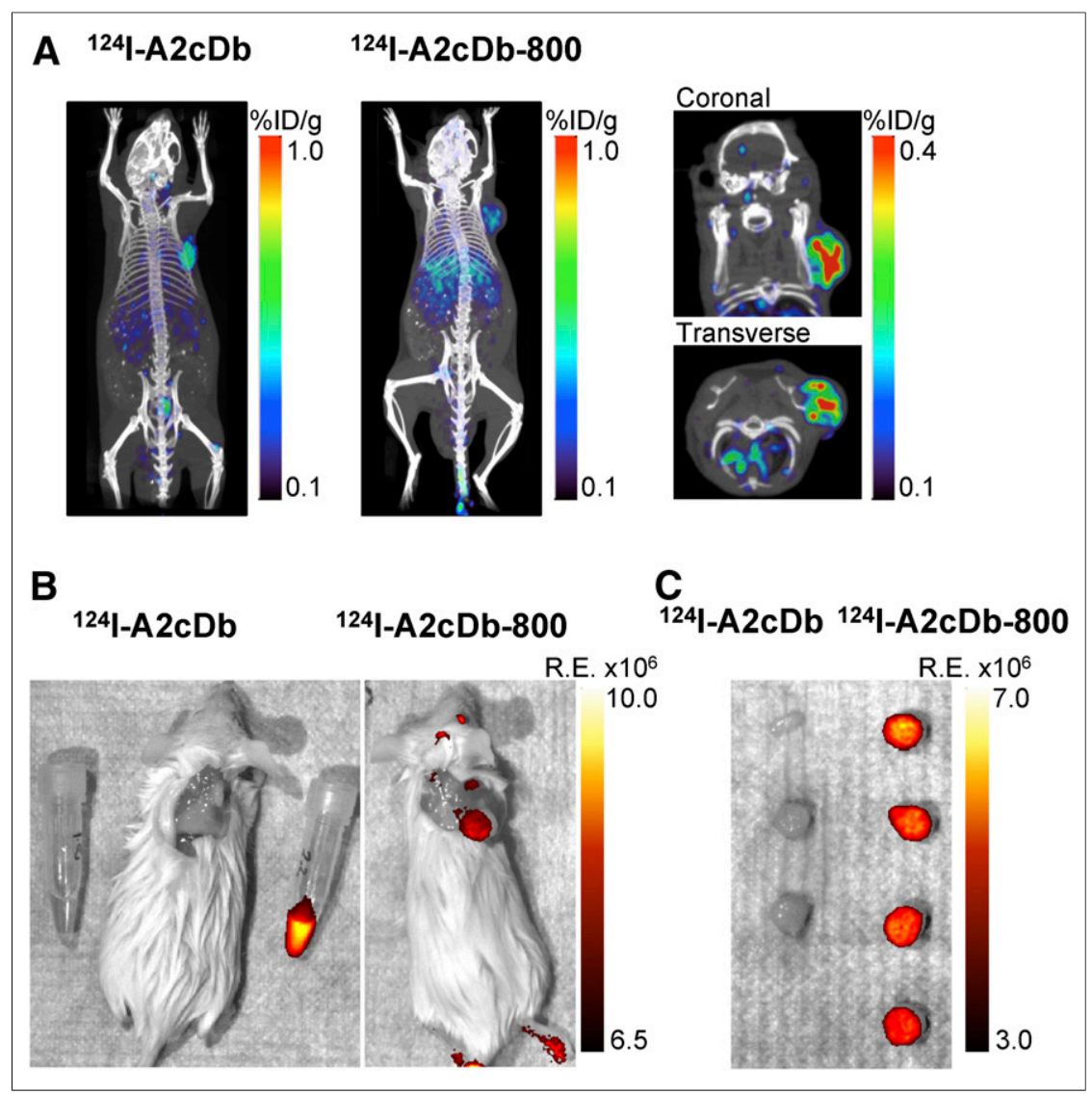

FIGURE 4. Dual-modality immuno-PET/NIRF imaging of PDX-PDACs. (A) ${ }^{124}$ l-immuno-PET comparing ${ }^{124} \mathrm{I}-\mathrm{A} 2 \mathrm{cDb}$ and dual-modality-labeled ${ }^{124} \mathrm{I}-\mathrm{A} 2 \mathrm{cDb}-800$ in PDX-PDAC (XWR6)-bearing NSG mice, $24 \mathrm{~h}$ after injection of $15 \mu \mathrm{g} / 2.96 \mathrm{MBq}$ of tracer. Depicted are representative scans (of $n=4$ ) as whole-body maximum-intensity-projection small-animal PET/CT overlays and 3-mm sections (coronal and transverse) of subcutaneous tumor. (B) Postmortem NIRF imaging of same mice with skin removed and PDX-PDAC in situ. (C) Resected xenografts ex vivo. R.E. = radiant efficiency.

\section{I-A2cDb-800 Dual-Modality Imaging of PDX-PDACs (XWR6)}

Immuno-PET comparing single-labeled ${ }^{124} \mathrm{I}-\mathrm{A} 2 \mathrm{cDb}$ and dualmodality ${ }^{124} \mathrm{I}-\mathrm{A} 2 \mathrm{cDb}-800$ in XWR6-bearing NSG mice was conducted $24 \mathrm{~h}$ after injection of $15 \mu \mathrm{g} / 2.96 \mathrm{MBq}$ of the respective tracers. Representative whole-body images (of $n=4$ ) show that the dual-modality probe ${ }^{124} \mathrm{I}-\mathrm{A} 2 \mathrm{cDb}-800$ successfully visualized the PSCA-expressing xenograft, with tumor uptake comparable to the single-labeled ${ }^{124} \mathrm{I}-\mathrm{A} 2 \mathrm{cDb}$ (Fig. 4A). Higher nonspecific uptake was seen in the liver, most likely because of the hydrophobic NIRF dye. The same mice underwent postmortem fluorescence imaging with the skin removed (Fig. 4B), and although mice injected with ${ }^{124} \mathrm{I}-\mathrm{A} 2 \mathrm{cDb}$ showed no fluorescent signal, a strong fluorescent signal was observed in the xenografts of mice injected with ${ }^{124} \mathrm{I}-\mathrm{A} 2 \mathrm{cDb}-800$. Low background uptake or autofluorescence was detected in the surrounding tissue. Tumors (Fig. 4C) and organs (Supplemental Fig. 1) were analyzed ex vivo to compare the relative fluorescent signal. Mice injected with ${ }^{124} \mathrm{I}-\mathrm{A} 2 \mathrm{cDb}$ showed normal autofluorescence in the stomach, intestines, liver, and spleen. In contrast, mice injected with ${ }^{124} \mathrm{I}-\mathrm{A} 2 \mathrm{cDb}-800$ showed fluorescent signal in the xenografts. Additionally, fluorescent signal was seen in the liver and kidneys. Ex vivo biodistribution ( $24 \mathrm{~h}$ after injection, Figs. 5A and 5B) confirmed similar uptake of both tracers in the xenografts, whereas nonspecific uptake in the liver, kidneys, and spleen was higher for ${ }^{124} \mathrm{I}-\mathrm{A} 2 \mathrm{cDb}-800$.

To summarize our results, use of the dual-modality anti-PSCA cys-diabody resulted in high-contrast immuno-PET/NIRF images of PDX-PDACs, suggesting that this probe might offer both noninvasive whole-body PET imaging to localize pancreatic cancer and NIRF imaging guidance to identify tumor margins during surgery.

\section{DISCUSSION}

Pancreatic ductal adenocarcinoma recurs at a rate of up to $80 \%$ within $2 \mathrm{y}$ of resection with curative intent. This high rate of recurrence is due to undiscovered metastases at the time of surgery or incomplete resection (positive margins) (7). The ability to noninvasively image pancreatic ductal adenocarcinoma and use intraoperative fluorescence guidance might improve diagnosis, staging, and resection.

We generated a dual-modality immunoPET/NIRF probe based on the humanized anti-PSCA $\mathrm{A} 2 \mathrm{cDb}$ by labeling $\mathrm{A} 2 \mathrm{cDb}$ with both IRDye800CW and ${ }^{124}$ I. The ability of the dual-modality ${ }^{124} \mathrm{I}-\mathrm{A} 2 \mathrm{cDb}-800$ to specifically target PSCA-expressing cells in vivo was confirmed by PET/NIRF imaging of human prostate cancer xenografts with high and low PSCA expression (22Rv1-PSCA and 22Rv1). Furthermore, ${ }^{124} \mathrm{I}-\mathrm{A} 2 \mathrm{cDb}-800$ was successfully used to image PSCA-positive PDX-PDACs, providing whole-body immuno-PET imaging that can visualize the tumor and NIRF imaging that can differentiate cancer from surrounding tissue.

Importantly, the engineered antibody fragment C-terminal cysteine enabled conjugation of IRDye800CW-maleimide away from the antigen binding site and without impairing diabody conformation or antigen affinity. The site-specific strategy limits the number of dyes per antibody fragment to less than $2: 1$, with the intention of preventing the dye from altering biodistribution or clearance of the antibody. Several groups have shown that higher degrees of labeling, structure, total charge, charge distribution, hydrophobicity, and plasma protein binding rate of the dye can lead to increased liver uptake and faster blood clearance $(20,22,34-36)$. The comparison of the dual-modality ${ }^{124} \mathrm{I}-\mathrm{A} 2 \mathrm{cDb}-800$ to the radiolabeled ${ }^{124} \mathrm{I}-$ $\mathrm{A} 2 \mathrm{cDb}$ in the 22Rv1-PSCA xenograft model confirmed that the NIRF dye did not affect in vivo antigen-specific tumor binding, biodistribution, or clearance. The molecular weight of the cys-diabody $(50 \mathrm{kDa})$ below the threshold for renal clearance, and the combination with the nonresidualizing ${ }^{124} \mathrm{I}$, result in low nonspecific background activity and make this targeting molecule favorable for imaging of the abdomen, especially considering the tendency of pancreatic cancer to metastasize to the liver. The short plasma half-life of the diabody enables same- or next-day immuno-PET imaging (37). At the same time, the IRDye $800 \mathrm{CW}$ is residualizing, 


\begin{tabular}{|c|c|c|c|c|c|c|c|c|c|c|c|}
\hline \multirow[t]{2}{*}{ A } & \multicolumn{5}{|c|}{$\% \mid D / g$} & \multirow{2}{*}{ B } & \multicolumn{5}{|c|}{ \%ID/organ } \\
\hline & 0.0 & 0.1 & 0.2 & 0.3 & 0.4 & & 0.0 & 0.1 & 0.2 & 0.3 & 0.4 \\
\hline Blood & & 口 & & & & Blood & & $\square^{\text {m }}$ & & & \\
\hline Tumor & & & & & & Tumor & ㅁㅁㅁ & & & & \\
\hline Heart & $\mathbb{1}^{\mathbb{1}}$ & & & & & Heart & i & & & & \\
\hline Lung & & (س & & & & Lung & 1 & & & & \\
\hline Liver & 1 & & प & & & Liver & . & & & II & \\
\hline Kidney & & 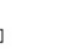 & Ш & & & Kidney & 1 & I & & & \\
\hline Spleen & & 四 & & 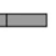 & & Spleen & 1 & & & & \\
\hline Stomach & & Ш & & & & Stomach & & $\square$ & & & \\
\hline Intestine & $1^{\prime}$ & & & & & Intestine & पा & & & & \\
\hline Muscle & i & & & & & Muscle & 1 & & & 124I-A2 & Db-800 \\
\hline Bone & 땀 & & & & & Bone- & 1 & & & $\mathrm{~A} 2$ & 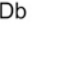 \\
\hline
\end{tabular}

FIGURE 5. EX vivo biodistribution $24 \mathrm{~h}$ after injection. PDX-PDAC (XWR6) tumors and tissues were harvested and $y$-counted (groups of $n \geq 3$ ). \%ID/g (A) and \%ID/organ (B) of each group are shown as floating bars (minimum to maximum, with a line at the mean).

and the lysosomal half-life of $2.33 \mathrm{~d}$ allows for longer detection (several days) of the fluorescent signal $(25,38)$.

Combining these two complementary imaging modalities into one dual-modality probe is attractive for seamless application and avoids differing biodistributions. In contrast, tracer cocktails, or separate conjugated tracers that no longer represent the original unlabeled antibody, are considered new and distinct medicinal products, requiring extensive testing and toxicology studies and complicating clinical translation.

$\mathrm{A} 2 \mathrm{cDb}$ has been previously used conjugated with the far-red fluorophore Cy5-maleimide for fluorescence-guided surgery in an intramuscular tumor model of 22Rv1-PSCA. After tumor resection under white light, positive surgical margins smaller than $1 \mathrm{~mm}$ were detectable by fluorescence and were confirmed by histology. Furthermore, the high specificity of $\mathrm{A} 2 \mathrm{cDb}$ for PSCA-expressing cells resulted in strong contrast with adjacent nerves, blood vessels, and normal tissues (29). In the present study, we used IRDye800CWmaleimide because it is produced under current good manufacturing practices, facilitating clinical translation, and because IRDye800CW (emission/excitation wavelengths, 774/789 nm) shows deeper tissue penetration and is less affected by tissue autofluorescence than Cy5 (emission/excitation wavelengths, 646/662 nm) (39).

Xenograft models that rely on highly homogeneous cell cultures generate reproducible data and are widely used to develop new tracers. However, one of their major limitations is that they do not reflect the complexity of human disease. Patient-derived xenografts better represent the tumors found in patients, but their heterogeneity makes them difficult to maintain and target for imaging studies.

Here, we screened PDX-PDACs for PSCA expression by immunoPET and found that all tested xenografts (5/5) expressed PSCA, although at varying levels. PDX-XWR6 showed PSCA expression in the lower range but could be detected by ${ }^{124} \mathrm{I}-\mathrm{A} 2 \mathrm{cDb}$ immuno-PET with high contrast. Even though PSCA expression in PDX-XWR6 seemed to decrease on subcutaneous passaging, the dual-modality ${ }^{124} \mathrm{I}-\mathrm{A} 2 \mathrm{cDb}-800$ successfully detected the xenografts by immunoPET, and subsequent NIRF imaging showed excellent delineation of the cancer tissue. Better imaging guidance during pancreaticoduodenectomy would have a tremendous impact on pancreatic cancer patients, given that median survival is vastly improved when a disease-free resection margin can be achieved (13 vs. 6 mo for tumornegative vs. tumor-positive margins) (40).

Future studies should include dualmodality imaging of clinically more relevant orthotopic PDX-PDAC models to ensure that PSCA-positive tumors are detectable when they grow tissue site-specifically and near the liver and spleen. Human-PSCA transgenic mice can be used to study biodistribution and off-target tracer uptake in the context of normal-tissue expression of PSCA. The dualmodality imaging approach should also be tested on antibodies against other biomarkers (41), because some pancreatic cancers do not express PSCA and therefore this marker would not be $100 \%$ sensitive.

\section{CONCLUSION}

This work showed specific targeting and high-contrast imaging of PSCA-expressing prostate cancer tumors and PDX-PDACs by immuno-PET and by NIRF imaging using a dual-modality tracer based on the anti-PSCA $\mathrm{A} 2 \mathrm{cDb}$ ( $\left.{ }^{124} \mathrm{I}-\mathrm{A} 2 \mathrm{cDb}-\mathrm{IRDye} 800 \mathrm{CW}\right)$. These encouraging results suggest that this dual-modality probe might offer both noninvasive whole-body PET imaging to localize pancreatic cancer and NIRF imaging guidance to identify tumor margins during resection. Clinical translation of this dual-modality tracer is facilitated by its being based on a humanized antibody fragment and conjugated to an NIRF dye intended for use in clinical trials.

\section{DISCLOSURE}

This work was supported by NIH grant R01 CA174294 and by UCLA Jonsson Comprehensive Cancer Center JCCC grant P30 CA016042. Timothy Donahue, Robert Reiter, and Anna Wu are members of the JCCC. Anna Wu is a founder, board member, and consultant to ImaginAb, Inc., and a consultant to Avidity Biosciences. Robert Reiter is a founder of ImaginAb, Inc. No other potential conflict of interest relevant to this article was reported.

\section{ACKNOWLEDGMENTS}

We thank Felix B. Salazar for technical assistance and Drs. Waldemar Ladno and Arion F. Chatziioannou at the UCLA Crump Institute for Molecular Imaging for help with PET and optical imaging.

\section{REFERENCES}

1. Ryan DP, Hong TS, Bardeesy N. Pancreatic adenocarcinoma. $N$ Engl J Med. 2014;371:2140-2141.

2. Bond-Smith G, Banga N, Hammond TM, Imber CJ. Pancreatic adenocarcinoma. BMJ. 2012;344:e2476.

3. Jha P, Bijan B. PET/CT for pancreatic malignancy: potential and pitfalls. $J$ Nucl Med Technol. 2015;43:92-97.

4. Wang XY, Yang F, Jin C, Fu DL. Utility of PET/CT in diagnosis, staging, assessment of resectability and metabolic response of pancreatic cancer. World J Gastroenterol. 2014;20:15580-15589.

5. Gioux S, Choi HS, Frangioni JV. Image-guided surgery using invisible nearinfrared light: fundamentals of clinical translation. Mol Imaging. 2010;9: 237-255. 
6. Smeenk HG, Incrocci L, Kazemier G, et al. Adjuvant 5-FU-based chemoradiotherapy for patients undergoing R-1/R-2 resections for pancreatic cancer. Dig Surg. 2005;22:321-328.

7. Kleeff J, Reiser C, Hinz U, et al. Surgery for recurrent pancreatic ductal adenocarcinoma. Ann Surg. 2007;245:566-572.

8. Reiter RE, Gu Z, Watabe T, et al. Prostate stem cell antigen: a cell surface marker overexpressed in prostate cancer. Proc Natl Acad Sci USA. 1998;95: 1735-1740.

9. Gu Z, Thomas G, Yamashiro J, et al. Prostate stem cell antigen (PSCA) expression increases with high Gleason score, advanced stage and bone metastasis in prostate cancer. Oncogene. 2000;19:1288-1296.

10. Han KR, Seligson DB, Liu X, et al. Prostate stem cell antigen expression is associated with Gleason score, seminal vesicle invasion and capsular invasion in prostate cancer. J Urol. 2004;171:1117-1121.

11. Zhigang Z, Wenlv S. Prostate stem cell antigen (PSCA) expression in human prostate cancer tissues: implications for prostate carcinogenesis and progression of prostate cancer. Jpn J Clin Oncol. 2004;34:414-419.

12. Argani P, Rosty C, Reiter RE, et al. Discovery of new markers of cancer through serial analysis of gene expression: prostate stem cell antigen is overexpressed in pancreatic adenocarcinoma. Cancer Res. 2001;61:4320-4324.

13. Katari UL, Keirnan JM, Worth AC, et al. Engineered $\mathrm{T}$ cells for pancreatic cancer treatment. HPB (Oxford). 2011;13:643-650.

14. Wente MN, Jain A, Kono E, et al. Prostate stem cell antigen is a putative target for immunotherapy in pancreatic cancer. Pancreas. 2005;31:119-125.

15. Saffran DC, Raitano AB, Hubert RS, Witte ON, Reiter RE, Jakobovits A. AntiPSCA mAbs inhibit tumor growth and metastasis formation and prolong the survival of mice bearing human prostate cancer xenografts. Proc Natl Acad Sci USA. 2001;98:2658-2663.

16. Wu AM, Senter PD. Arming antibodies: prospects and challenges for immunoconjugates. Nat Biotechnol. 2005;23:1137-1146.

17. Wu AM. Antibodies and antimatter: the resurgence of immuno-PET. J Nucl Med. 2009;50:2-5.

18. Knowles SM, Wu AM. Advances in immuno-positron emission tomography: antibodies for molecular imaging in oncology. J Clin Oncol. 2012;30:3884-3892.

19. Wu AM. Engineered antibodies for molecular imaging of cancer. Methods. 2014;65:139-147.

20. Boswell CA, Tesar DB, Mukhyala K, Theil FP, Fielder PJ, Khawli LA. Effects of charge on antibody tissue distribution and pharmacokinetics. Bioconjug Chem. 2010;21:2153-2163.

21. Boswell CA, Mundo EE, Zhang C, et al. Impact of drug conjugation on pharmacokinetics and tissue distribution of anti-STEAP1 antibody-drug conjugates in rats. Bioconjug Chem. 2011;22:1994-2004.

22. Conner KP, Rock BM, Kwon GK, et al. Evaluation of near infrared fluorescent labeling of monoclonal antibodies as a tool for tissue distribution. Drug Metab Dispos. 2014;42:1906-1913.

23. Te Velde EA, Veerman T, Subramaniam V, Ruers T. The use of fluorescent dyes and probes in surgical oncology. Eur J Surg Oncol. 2010;36:6-15.

24. Vahrmeijer AL, Hutteman M, van der Vorst JR, van de Velde CJ, Frangioni JV. Image-guided cancer surgery using near-infrared fluorescence. Nat Rev Clin Oncol. 2013;10:507-518.
25. van Dam GM, Themelis G, Crane LM, et al. Intraoperative tumor-specific fluorescence imaging in ovarian cancer by folate receptor-alpha targeting: first inhuman results. Nat Med. 2011;17:1315-1319.

26. Harlaar NJ, Koller M, de Jongh SJ, et al. Molecular fluorescence-guided surgery of peritoneal carcinomatosis of colorectal origin: a single-centre feasibility study. Lancet Gastroenterol Hepatol. 2016;1:283-290.

27. Lamberts LE, Koch M, de Jong JS, et al. Tumor-specific uptake of fluorescent bevacizumab-IRDye $800 \mathrm{CW}$ microdosing in patients with primary breast cancer: a phase I feasibility study. Clin Cancer Res. 2017;23:2730-2741.

28. Liu K, Lepin EJ, Wang MW, et al. Microfluidic-based ${ }^{18} \mathrm{~F}$-labeling of biomolecules for immuno-positron emission tomography. Mol Imaging. 2011;10:168176.

29. Sonn GA, Behesnilian AS, Jiang ZK, et al. Fluorescent image-guided surgery with an anti-prostate stem cell antigen (PSCA) diabody enables targeted resection of mouse prostate cancer xenografts in real time. Clin Cancer Res. 2016;22:1403-1412.

30. Lepin EJ, Leyton JV, Zhou Y, et al. An affinity matured minibody for PET imaging of prostate stem cell antigen (PSCA)-expressing tumors. Eur J Nucl Med Mol Imaging. 2010;37:1529-1538.

31. Knowles SM, Zettlitz KA, Tavare R, et al. Quantitative immunoPET of prostate cancer xenografts with ${ }^{89} \mathrm{Zr}$ - and ${ }^{124} \mathrm{I}$-labeled anti-PSCA A11 minibody. J Nucl Med. 2014;55:452-459.

32. Loening AM, Gambhir SS. AMIDE: a free software tool for multimodality medical image analysis. Mol Imaging. 2003;2:131-137.

33. Verde F, Fishman EK. Calcified pancreatic and peripancreatic neoplasms: spectrum of pathologies. Abdom Radiol (NY). 2017;42:2686-2697.

34. Sampath L, Kwon S, Ke S, et al. Dual-labeled trastuzumab-based imaging agent for the detection of human epidermal growth factor receptor 2 overexpression in breast cancer. J Nucl Med. 2007;48:1501-1510.

35. Cohen R, Vugts DJ, Stigter-van Walsum M, Visser GW, van Dongen GA. Inert coupling of IRDye800CW and zirconium-89 to monoclonal antibodies for single- or dual-mode fluorescence and PET imaging. Nat Protoc. 2013;8:10101018.

36. Cilliers C, Nessler I, Christodolu N, Thurber GM. Tracking antibody distribution with near-infrared fluorescent dyes: impact of dye structure and degree of labeling on plasma clearance. Mol Pharm. 2017;14:1623-1633.

37. Wu AM, Yazaki PJ. Designer genes: recombinant antibody fragments for biological imaging. Q J Nucl Med. 2000;44:268-283.

38. Cilliers C, Liao J, Atangcho L, Thurber GM. Residualization rates of nearinfrared dyes for the rational design of molecular imaging agents. Mol Imaging Biol. 2015; 17:757-762.

39. Adams KE, Ke S, Kwon S, et al. Comparison of visible and near-infrared wavelength-excitable fluorescent dyes for molecular imaging of cancer. J Biomed Opt. 2007; 12:024017.

40. Evans DB, Farnell MB, Lillemoe KD, Vollmer C Jr, Strasberg SM, Schulick RD. Surgical treatment of resectable and borderline resectable pancreas cancer: expert consensus statement. Ann Surg Oncol. 2009;16:1736-1744.

41. Luo H, England CG, Goel S, et al. ImmunoPET and near-infrared fluorescence imaging of pancreatic cancer with a dual-labeled bispecific antibody fragment. Mol Pharm. 2017;14:1646-1655. 